\title{
Quantum Interactions as Niche-Structure
}

\author{
Michael J. Pocklington \\ Department of Genetics, University of Leicester, LE1 7RH, United Kingdom
}

\begin{abstract}
In the field of systems biology, the molecular interactions constituting the life of organisms may be represented in interaction maps. The genetic interaction map is unique because it concerns only the interactions of an organism, occupying its niche, producing biological fitness. The procedure for obtaining this map can be idealized. Underlying the map for any occupied niche there must exist a temporally closed (developmental) informationprocessing structure, or causal plexus. This must be located within a temporally open (evolutionary) niche-structure, or causal nexus. The framework allows simplification naturally, identifying molecules and sub-molecular particles also as occupied niches. Quantum interactions are then revealed as the merging and splitting of niches, the flux determined by the entropy gradient. Across all levels of the self-similar niche-structure continuum, occupied niches determine the construction of empty niches, and empty niches provide the potential for the evolution of the occupied niche-structure.
\end{abstract}

Keywords: quantum interaction, niche, niche-structure, natural genetic interaction map, imaginary chemostat, causal plexus, causal nexus.

\section{Introduction}

In this paper I wish to draw attention to an increasingly popular visualization technique in the field of systems biology, the interaction map. There are many different types of interaction map, but the genetic interaction map is special. It visualizes the network of interactions constituting organismic function using information gathered from genetic mutations. It does this using only one kind of measurement: the measurement of fitness. This is a map of interactions defining exactly those functions yielding the survival of the organism. Here, we find a way to generalize it.

In its experimental production, the genetic interaction map emerges from pairwise combinations of mutations in genetically tractable micro-organisms. When they are introduced into the same cell, two mutations are seen to be functionally independent if the double mutant has a fitness deficit that is the expected product of the mutations acting alone. A genetic interaction refers to the case giving an unexpected fitness measurement against the aforementioned background. For example, two minor mutations, placed together in the same gene, could unexpectedly cause the gene product to catastrophically fail. On the other hand, two serious mutations placed together in a gene could unexpectedly compensate for each other. The interaction shows that the mutations have some functional relationship to each other; in this example, physical proximity in a gene product such as a protein, which could be mis-folded. 
Genetic interactions between pairs of mutations are not limited to co-location in the same gene. This is because the molecular activity of the cell is embodied in space; it is organized in pathways and physical structures which are encoded by separate genes. Mutations affecting adjacent products in pathways, or contained in structures in physical contact, will reveal interactions linking separate genes. This is both the theory, and the observation. Very large numbers of pairwise interactions of mutations, combined into a map, may usefully depict every genetically-determined function of an organism. This is the genetic interaction map (see Fig. 1; [1]). It is not to be confused with other types of interaction map, or with genetic regulatory networks.

The earliest interaction maps were drawn by Benzer [2], when they were called genetic complementation maps. There was some early discussion concerning the philosophy underlying the interaction map itself; but then, as now, interest was directed at the content of the map. We shall not be interested in the content of the map. We are interested in what sorts of entities can yield such a map, and what the map actually means, i.e. the type of territory that the map depicts.

In order to address such questions, we will employ a thought-experiment. We will use existing knowledge to recursively define and idealize the procedure for obtaining the map. This gives the natural genetic interaction map, which is unique and perfect for every organism. Because it is perfect, it can never be completely achieved other than by the idealized method outlined here. The natural genetic interaction map may be likened to the square root of 2 , which can not be expressed as a precise numerical value, but can be perfectly defined by an exact procedure.

In the laboratory, an artificial environment known as a chemostat is used to obtain the fitness data for the map. In this paper, an imaginary chemostat is the idealized method for determining fitness. Properly constructed, the imaginary chemostat must produce a fitness measurement that is a probability per unit time that the mutant organism will be lost from the chemostat. Significantly, this measurement of fitness is not constrained by ideas about what constitutes an "organism". Entities other than classical organisms could maintain themselves in a chemostat. Similarly, the idea of a "gene" is here superseded by an operational definition. Any defect, heritably transmitted by any entity, could have its fitness consequences measured, and would yield a map. In biological organisms, this includes epigenetic information. Heritable transmission outside of canonical genetics is found in realms other than organisms, such as systems of human organization and production, containing such things as programs, propositions, procedures, protocols, plans, concepts, and customs. Heritably transmitted information is also contained in realms lacking conspicuous symbolic content, such as those described by chemical or electronic feedback loops and networks. Examples of the latter are communication networks and brains.

This method of visualization suggests a single extended framework for understanding nature that is not merely a unity of representation. Not only are niche-filling entities conspicuous by their abundance in nature, but they are linked in ecosystems that may be continuous across different realms. Here we extend the framework from biological organisms downwards to entities in the quantum realm. The framework does not suffer from a classical provenance, and has much in common with quantum theory, but the details of the relationship have yet to be worked out. 


\section{Construction of the Genetic Interaction Map}

Recent advances in automated high-throughput technology and software techniques have allowed the construction of interaction maps for free-living organisms (Fig. 1; [1]). Construction of genetic interaction maps relies on the expectation that if two mutations are functionally independent, the fitness phenotype of the double mutant would be expected to be the product of the measured fitness phenotypes of the two mutants. Suppose we have two functionally independent mutations, each with a fitness of 0.5 relative to the strain without a mutation (the "wild-type"). Then if the two functionally independent mutations are introduced into the same genetic background, the relative fitness of the double mutant is expected to be 0.25 . Deviations from this expectation imply the existence of functional associations that embody the life of the organism. Here is not the place to enumerate the various ways in which mutations can interfere with each other to produce the deviations seen, but the interested reader may find useful [1] and [3] and the references contained therein. Be aware that the classical study of "epistasis", which holds genetic interactions as its subject of interest, tolerates many mathematically courageous formulations that are not relevant to our current analysis.

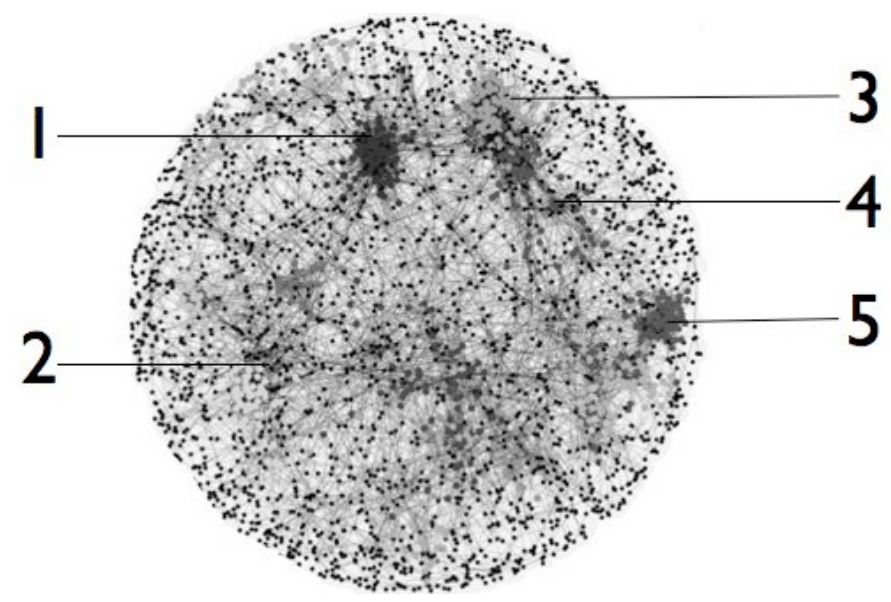

Fig. 1. The genetic interaction map for yeast Saccharomyces cerevisiae, redrawn from [1]. Each node (dot) represents a gene, but each gene could in turn be comprised of thousands of nodes, each representing a single mutation. Clusters of nodes show interacting genes, participating in identifiable cellular functions; here there are shown five labelled examples: 1, secretion; 2, ribosomal functions; 3 , glocosylation and cell wall biosynthesis; 4, cellular morphogenesis; 5, DNA replication and repair.

The only phenotypes requiring to be measured are the fitnesses of the single mutants and all the double mutants (pairwise combinations of single mutations in the same organism). This is routinely achieved using a variety of methods in the highthroughput laboratory. Low resolution measurements of fitness can be obtained from growth rates in liquid medium, but the archetypal measurement of fitness with the greatest sensitivity is to grow each mutant together with the wild-type strain under 
competition in a chemostat. A chemostat allows the continuous culture of organisms under essentially constant conditions. It comprises a culture vessel through which there is a continuous defined flow of nutrient medium. Provided the flow rate of the medium does not exceed the growth rate of the organism, the organism will survive in the chemostat, and essentially constant conditions are obtained. "Essentially constant" means that averaged over the duration of many inhabited chemostats of the same type, overall conditions will have a constant distribution i.e. there is a definable state space in the sense of dynamical systems theory.

With a few well-known and rare exceptions, the wild-type strain will eventually displace the mutant strain in the chemostat, and the time taken for it to do this is the measure of the (lack of) relative fitness in the mutant. Precision in the measurement of fitness can be increased by repeated measurements in the rigorously-standardized chemostat. There is no indication to suggest any value in combining together more than two mutations. This is because we wish to obtain a map, for which pairwise interactions are sufficient. The organism under study needs to be genetically pure except for the mutations in question.

Graphical techniques can incorporate fitness data for all the mutants represented as nodes in a static graphical plot. This can be achieved in a variety of ways in practice, but for our purposes we need only imagine one simple method. For each pairwise mutant combination, the deviation from expected fitness is a quantitative relationship between two mutants. This quantity can be used to establish an edge length between each pair of nodes, by taking the mean edge length between all the nodes and adjusting it for each pair of nodes, bringing two nodes closer together if they show an interaction. Pairs of mutations showing a large deviation in fitness will assume a position in close proximity to each other, provided other mutation-combinations allow it; whereas pairs of mutations showing no deviation in expected fitness will come to occupy positions on opposite sides of the map, were it not for connections with other mutations. The genetic interaction map in Fig. 1, redrawn from reference [1] illustrates a similar visualization method to the one described above.

Specific environmental perturbations (changes in the nutrient medium or chemostat parameters) can be contrived, producing nodes on the map representing environmental conditions that will connect to the nodes representing genetic elements involved in dealing with those conditions. The network of interactions therefore reaches out into the environment, to include nutrients, toxins, and physical conditions.

\section{The Imaginary Chemostat}

Recall the measurement of fitness, in which two types of organism are grown together in the laboratory chemostat until only type one remains. The procedure can be repeated until a statistically relevant result is obtained. Since we know the map depends for accuracy on an arbitrarily large number of runs of the chemostat, let us imagine being able to make a very large number of runs, say $10^{100}$ for each mutant. The chemostat conditions for the runs would have to be standardized. We would expect the 
interaction map to sharpen up indefinitely; but we could expand the view of the map and continuously inspect it for greater detail. Since we would now be making an impossible number of measurements, we might just as well free the chemostat from any other practical limitation, such as size and shape of containing vessel, and constituents of the medium flowing through the chemostat.

For the measurement of fitness it is necessary that an organism must not mutate, in other words, we must be able to stabilize (or "freeze") genetic information. This is a requirement for holding constant the object of inquiry.

We need not assume that genetic information must be carried by DNA, because we could collect every different mutant that could ever occur in our organism by waiting until such a mutation were to occur; and then stabilize it. A mutation identifies an inherited trait. If an inherited trait could be carried by self-replicating RNA, or a methylation pattern independent of the DNA base sequence, or a prion, we could imagine stabilizing it, isolating the strain of organism carrying it, and measuring its fitness in the same way as for any other kind of mutant. Since we have fully defined the environment in which the organism survives, any mutation, and hence functional genetic information, is operationally defined by its effect on fitness. If it has an effect on the life of the organism, it will appear as a node on the map.

\section{What the Genetic Interaction Map Means}

As is well known, the map and the territory are not the same thing. In its construction, the genetic interaction map is a static graphical plot of failures in fitness due to mutant gene combinations; so what, exactly, is the territory? It appears intuitively obvious that the map refers to a network structure describing all the molecular activities of the organism; indeed, the integrity of the entire field of molecular biology rests on the existence of such a structure. An underlying functional network may be held in the mind of the biologist (for examples, see refs [4-6]) but only within the context of experiments and contrived observations, or in some other way disconnected from the selecting environment. Here, we identify it as the whole functional network corresponding to the survival-strategy of the real organism, naturally selected in its real environment. It must be a unitary object because, insofar as we can freeze the genetic information that defines the organism, the map is singular and precise, and it must therefore be temporally closed. In other words, we must have accounted for all the possibilities for the developmental trajectory of the network. Since we understand that the network depicts, for a genetically-defined organism, the causal flow of information, energy and materials, we may call it a causal plexus. This distinguishes it from the wider, temporally open, causal nexus. From our present perspective the causal nexus is the network containing a diverse assemblage of genetically-defined organisms in their respective niches within the niche-structure (ecosystem) where evolution takes place. It may be observed at this point that the ecological niche-structure bears a resemblance to, as well as continuity with, the causal plexus that defines the organism. 


\section{Conceptual Simplification}

With only one kind of measurement, the genetic interaction map yields an ideal image of an organism, in the form of a map; we have seen that this must refer to an underlying network of molecular activity, here called a causal plexus. It has also given a precise conception of an environmental container, or niche, which we can construct in principle, called an imaginary chemostat.

Here we aim to conceptually simplify the system, perhaps to reveal its fundamental nature. This process of conceptual reduction I will call reductio ad extremum. Starting with an organism such as yeast, we could grow it in increasingly permissive (that is, less demanding) imaginary chemostat environments. Then, we could delete the unnecessary genes, giving us a simpler type of organism, and a simpler map. We could delete the genes encoding metabolic enzymes, because we could supply their products in the chemostat medium. Functions such as sporulation, sexual reproduction, stationary phase, aerobic respiration, and much regulatory activity would be redundant in a suitable chemostat, and the genes encoding these functions could be deleted. We would still have an organism, a niche, a map, and an underlying causal plexus.

Continued survival of an entity in the chemostat need not rely on cellular replication, since continuous occupation of the chemostat vessel could be achieved by the growth of hyphae, that is, without cellular separation. A cellular structure involving membranes is not required, since a structure such as a polymeric molecule could propagate in the chemostat. We could delete the genes encoding the system for energy metabolism, but then we would have to supply (for example) ATP in the chemostat medium, otherwise the individual entities would not be able to grow. However, if the entity was simple enough, we would not need an energy currency such as ATP; instead, we could rely on a supply of energetically activated precursors for building the structure. Expressed more generally, an entropy gradient would seem, at this point, to be required.

After many rounds of reductio ad extremum we would be left with a simplified organism, and a simplified interaction map (Fig. 2). Eventually, we would arrive at either of two possibilities.

In the first possibility, we could follow the process of reductio ad extremum to the point where the entity in the imaginary chemostat would assemble spontaneously from the precursors supplied in the chemostat medium. Inoculation of an existing entity would not be necessary. We could separately make defined changes ("mutations") to the medium entering the chemostat, and likewise to the entity in the inoculum. This would yield a genetic interaction map in which all the "mutations" would map to the chemostat itself (the medium, or the vessel walls). "Mutations" made to the entity in the inoculum would make no difference to the presence of the entity in the chemostat; they would not be represented on the map. We could call the entity a nonsicle (non-cycle), and we could call the highly contrived medium an "assertive" medium.

In the second possibility, occupation of the chemostat would depend upon an initial inoculation with a niche-filling entity. In this case, it would indeed be possible to pro- 
duce an interaction map for the inoculated entity. We could call the chemostat environment a "permissive medium". A familiar example is to be found in the commonlyused polymerase chain reaction (PCR). Here, the permissive medium supplies energetically-activated monomers, polymerase enzymes, pulses of heat to allow separation of the hydrogen-bonded strands, and a suitable aqueous environment. We could usefully hold a generalized conception of a PCR-like reaction for open-ended propagation, while recognizing that self-templating molecules need not be limited to nucleic acids. After this fashion, a large variety of simple self-reproducing physical entities could be propagated in an imaginary chemostat, since the medium, physical activity, and vessel walls of the imaginary chemostat are not limited in any way.

We could obtain interaction maps for crystals, micelles, hydrophobic complexes, or propagating molecular agglomerations generally (agitation or heat pulses will be assumed). All that is necessary is that the entities will fill a chemostat by growth from an inoculum, thereafter to propagate and survive. We can imagine other well-known self-maintaining entities, such as those generally described as dissipative structures: a non-exhaustive list would include flames, corrosions, vortexes, avalanches, and dustbunnies. We expect to be able to construct imaginary chemostats, and derive interaction maps for all of the aforementioned entities.

The functional networks underlying the very simplest physical entities in the examples above could be described as limit-stable positive feedback loops, or state-cycles (Fig. 2). These state-cycle "organisms" will have few-node interaction maps, perhaps corresponding to the sites for addition of subunits, or sites of physical fragility.

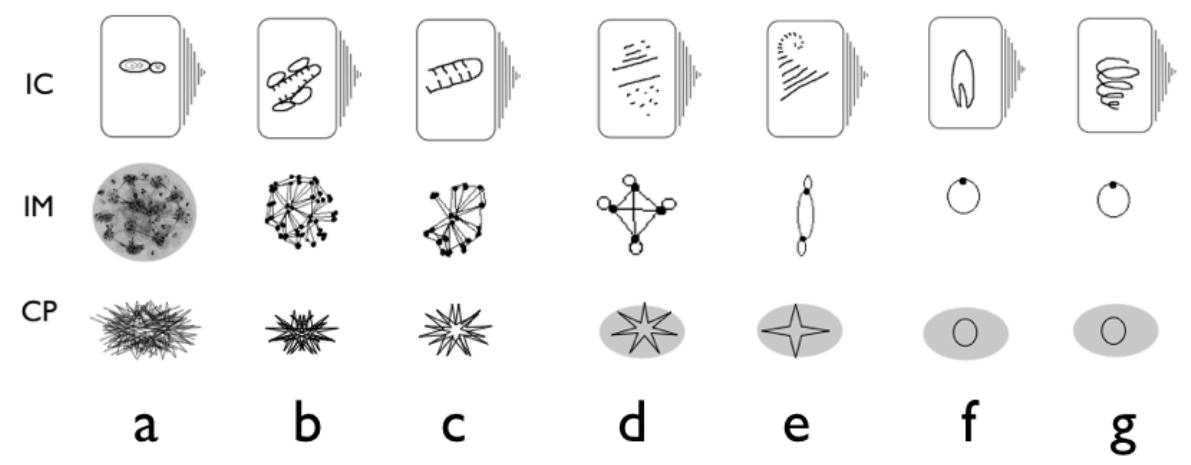

Fig. 2 Examples of entities in the conceptual simplification. Left to right, increasingly permissive medium. IC, imaginary chemostat. IM, imagined genetic interaction map. CP, imagined causal plexus. a, yeast. b, ribonucleoprotein complex. c, nucleic acid. d, self-templating polymer or crystal. e, non-templating polymer growing by terminal addition of subunits. $f$, flame. $g$, vortex. 


\section{The Molecule as an Occupied Niche}

Consider the case of a population of entities such as crystals or polymeric molecules, as described above, propagating by cycles of breakage and growth within a permissive medium, with the potential to indefinitely occupy the imaginary chemostat. We could ask how many individual molecules there would have to be in the chemostat to qualify as a replicating entity. Measurement of survival in the imaginary chemostat requires only that an organism or entity can be reliably observed. We are forced to conclude that to qualify as an entity occupying the chemostat, we do not need a population larger than one (or two, depending on the stage of replication).

Thus the entity occupying the niche could be a single polymeric molecule, replicating by a cycle of growth and fragmentation, in a suitable permissive medium. Let us now ask if we can simplify it further. Observing a cycle of polymerization and breakage, we can reduce this conceptually to a dimer. In other words, the entity inhabiting the imaginary chemostat is now a dimeric molecule, dissociating into monomers under the conditions provided by the permissive medium, such that each monomer is then available for attachment to an activated monomer, again provided by the permissive medium, eventually forming a new dimer. The cycle of "reproduction" would continue, and the chemostat would be filled. We continue to see an entity in a niche; we could obtain "mutants", and construct an interaction map.

This can be simplified even further without departing from the conceptual framework. The entity occupying the chemostat does not have to be a homodimeric molecule; it could be a heterodimer. How large does the smaller subunit of the heterodimer have to be, to qualify for restoring the entity to a "mature" form? It could be a single atom, say of oxygen; and thus our entity could be maintained indefinitely through cycles of oxidization-reduction in a suitable chemostat. But why restrict our notion of dynamic identity to oxidation-reduction cycles? Why not maintain identity through addition and removal of a single electron? Or a photon?

If it was a photon, we would now have reduced the entity inhabiting the imaginary chemostat, originally undeniably an organism within a niche, to the size and complexity of a single excitable molecule propagating through time in a permissive medium that supplies an energy gradient.

Surely, "niche-filling" does not reduce to excitability? After all, a single stable molecule in the ground state is hardly any different from its dynamically excited counterpart, and it, too, "propagates" through time, by virtue of its internal sub-molecular interactions. We should therefore consider the dynamically excited molecule and its ground-state counterpart as separated by a step in appearance, but not a jump in realm.

In either case, the imaginary chemostat is not now a container; instead, it is a localized arrangement of energy (a container of sorts) where the positional information in the trajectories of the sub-molecular particles in the molecule itself constitute selfmaintaining structural information. Note that the imaginary chemostat continues to function as before: as a niche-filling entity, the molecule does have a measurable "fit- 
ness", since it could (and inevitably would) disintegrate with some non-zero probability, were we to "mutate" positional information carried by atoms. We can draw an interaction map, by "mutating" the molecular structure, now interfering with the energy states of electrons, atoms, and perhaps other particles, thereby changing the fitness (stability) of the molecule.

The thought-experiment of reductio ad extremum may seem trivial, but the purpose is to establish an unbroken chain of identity while simplifying the organism-plus-niche system. The outcome is perhaps not surprising: through the imaginary chemostat we establish the conceptual continuity of a niche occupied by an organism defined by boundaries at the ecological scale, with a niche occupied by a molecule defined by boundaries at the molecular scale. The framework links the thermodynamic energy well of the ecological niche occupied by the organism, with the quantum electrodynamic energy well occupied by the molecule. There is no discontinuity in the reality, only in the construction that has entered social familiarity.

This is not a process of drawing analogies, although unintended and unhelpful analogies may be drawn. Since the reductio ad extremum is an unbroken series, it indicates that the structure underlying the occupied ecological niche is the same type of object as the structure underlying the molecule, the thing here called the causal plexus.

It is likely that any or all of the above features of niche-filling entities could be encompassed in some kind of taxonomic structure. The current framework is not constrained by taxonomic considerations. Instead, it draws attention to a type of structure that demands a taxonomy. Such a taxonomy, though useful, could make no difference to the underlying reality.

Any suggestion that there is more than one type of natural entity in the chain from molecule to organism must suggest a stage in the proposed sequence of reductio ad extremum at which one type of entity becomes another. For example, proposed nichefilling entities that have not been shown to exist in nature (such as replicating molecules) might be dismissed as examples of "real" entities, thereby disallowing the reductio ad extremum to continue beyond this point, and imposing an early discontinuity in the type of entity. However, this suggestion would continually have to be updated to take into account the discovery of additional simple self-maintaining systems.

Or, it could be suggested that the unique possession of "genetic information" by an entity could identify the end-point of one type of entity and the beginning of another. But in that case, how much genetic information would qualify, and how often would it have to be used? What exactly is genetic information anyway, if it is not just information allowing a repeatably observable state? A growing crystal, dividing by regular agitation of the medium or a heat pulse, may contain impurities that promote its survival. Do these impurities not constitute genetic information, if they are stably transmitted? Perhaps only templated information could qualify as "genetic", while other types of (positional) information would be discounted, revealing a discontinuity in the reductio ad extremum? These distinctions would appear to be arbitrary. 
Or, perhaps it may be thought that a flux of energy, absent from a system merely maintaining molecular identity but required for self-maintenance in complex organisms, could identify a fundamental discontinuity in the type of entity. However, in that case, a dynamically driven excitable molecule could be viewed similarly to an organism, and should be admitted to have an underlying causal plexus; whereas the same molecule in the ground state, with a mappable internal structure, would not. Again, this would be an arbitrary distinction.

Thus we find that the framework provided by the imaginary chemostat does not suffer from the need to draw a discontinuity in the spectrum of complexity from the organism down to the molecule. It would recognize a discontinuity if such a discontinuity was found (as in the example of the nonsicle, in which the entity occupying the niche has an empty interaction map); but all the entities described above, from complex organisms down to molecules, have an equivalent type. They are all self-maintaining, niche-filling entities for which we can, in principle, derive an interaction map, in turn pointing to an underlying functional network, here called a causal plexus.

It is reasonable to suggest that if we can arrive at a molecule from an organism by reductio ad extremum, then it is trivial to extend this framework further downwards to include atoms and subatomic particles; and it would be unnatural not to proceed with such an extension. This is because not only molecules, but all the components that make up a molecule, ultimately fermions and bosons, propagate through time in the same way relative to the "bottom level" permissive medium. This universality of behavior for all entities at the level of the molecule and below is expressed in the usage of the quantum theory to deal with these entities. We may identify the permissive medium as the level at which Planck scale interactions occur.

In ecological niche structures, the process by which one niche is successfully invaded by organisms from another may be called natural selection. We routinely visualize this by imagining a series of connected niches in a niche structure, such as an evolutionary series of yeast evolving to exploit a novel sugar. The change in the genetic makeup of the yeast is an example of downward causation, caused by natural selection for survival of novel mutants in successive niches. I will suggest that the same process explains the selection of empty niche structures in the permissive medium, and that this in turn constrains the propagation of particles through the permissive medium.

\section{Discussion}

Application of the imaginary chemostat revealed a conception of "the organism" not as an individual, or a population, or a species, or as disembodied behavior, but as the occupied niche. We have found that the occupied niche is a fundamental entity, not limited to populations of organisms; and it deserves a special name, here called the causal plexus. The imaginary chemostat was the tool of thought used to arrive at this fundamental entity, and to extend a framework encompassing instances of it, down to the quantum realm. I will argue elsewhere that this framework must encompass all of 
reality, including such things as human organizations, minds, and structures such as mathematics.

The occupied niche revealed in this way is a singular object. Even without extending the line of reasoning down to the quantum realm, we can see that its singular nature makes it a quantum object. This is because of the way it is constructed. The growth of organisms demands the definition of a container which exactly describes all of the things it could be; and an organism defined ultimately by survival. The measurement of survival, equivalent to fitness, repeated many times, can become as precise as we like, thus yielding the singular object. Note that it is a conceptual object. Real organisms we see in the world around us are particular instances of this object. However similar they may be in appearance to each other in genetic definition and environmental provenance, they will always be distinguishable by the method described. This means that different individuals within a species, for example, will not occupy exactly the same niche, but a "niche-cloud". Species have found a way to exploit nicheclouds through the generation and maintenance of appropriate variability.

But let us stay with the singular, conceptual object, the occupied niche. As well as having a singular nature, it is "temporally closed"; it is also dynamically selfrenewing, provided it does not interact with another system. If it interacts outside of its definition as described, it has been changed; we are no longer looking at the same object, and any further behavior is unpredictable. Its envelope or interface with the environment is part of its (self-renewing) functional network; it has zero-order interaction kinetics; and it can form new stable structures by engaging with other niches, forming a niche-structure. Interactions take the form of binary fusion, or they can disintegrate into a product-pair, the directionality provided by natural selection. The foregoing is a description of niches occupied by biological organisms, as revealed using the imaginary chemostat (sections 4 and 5); yet it could also be a description of quantum particles. The explanation offered here for this similarity of description is that they are, indeed, the same type of object.

Interactions between them must also be of a single type. Consider the constitution of "the organism" as an occupied niche. It is shown (sections 4,5 , and 6 above) to be a functional network of molecular reactions. But the framework reveals molecules themselves to be occupied niches, so the molecular reactions within an organism itself must define a niche-structure. Thus the framework as a whole describes a single, selfsimilar niche-structure. There are no absolute levels of organization spanning the niche-structure, since organisms not only eat each other, they also eat molecules (or, indeed, photons) directly. Thus a level of organization may be a pedagogical convenience, but a more mature viewpoint sees just one, big, self-similar niche-structure.

According to this niche-filling conception of reality, stable niche-particles renew themselves because on this scale of existence there is nowhere else for energy or information to come from, or go. We could acknowledge the notion of a Hamiltonian system, but here we need only care about a defined system of occupied and empty niches each with a precisely allowed quantity of energy for each niche-particle type. Since niches are tightly defined in themselves, information and energy must be conserved when changes in niche occupancy occur. This is entirely consistent with an ecological niche-structure in which the flow of information or energy must be com- 
pletely accounted for, and at least in principle, all the occupied niches identified, and their sources of energy and information, traced.

A particle has to take some definite identity, even though this might be of a distributed form. We are familiar with niches being distributed in space, so we should have no difficulty in thinking of an occupied niche that is a fundamental particle (nicheparticle) as a particle's -worth of energy, perhaps also having internal positional information giving it a type-identity. All it can do is invade niches that allow it to maintain its identity, since it has an ideal nature; all energy and information must be accounted for. It could enter a neighboring empty niche, maintaining its particular identity; or, if the neighboring niche is already filled, it could (probabilistically, depending on empty niche availability) merge its information with it, creating a new type of niche-particle with a type-identity embracing both of the niche-particles that contributed to it. We would identify this as an interaction. Run the interaction in reverse to allow the niche-particle to disintegrate. The entropy gradient provides directionality by supplying or reducing the activity of entities at either end.

Returning to the bottom-level permissive medium, let us suppose that the current state of reality comprises niche-particles propagating through time and space, where the permissive medium comprises only empty niches. Where might these empty niches come from? Recalling that niches are like islands in time, it could be suggested that the empty niches in the permissive medium are closed time-symmetrical causal loops, perhaps identical to the structures visualized by Feynman diagrams. We could further suppose that these structures could be chained together over arbitrary distances in space, provided that they never violate closed time-symmetrical causality. Empty niches could terminate at an occupied niche-particle, thus providing an opportunity for niche-particle time-evolution (i.e. to probabilistically invade the empty niche); assuming the empty niche structure as a whole must always conform to closed timesymmetrical causality.

This would be possible if the causal loops were allowed to grow from nascent seeds or bubbles. These could join up together, constructing an empty niche-structure having an indeterminate spatial extent, through which a niche-particle could propagate. In this case, the empty niche-structure (a template of "virtual particles") could be seen as naturally selected by the current state of all real niche-particles. We could say that the empty niche-structure is downwardly caused by the current occupied niche-structure.

Since time is entirely constituted by change at the niche-particle level, the rate of change of the underlying permissive medium in creating the empty niche structure has no meaning, i.e. it is extra-temporal. Seen from the vantage point of occupied niches, any change in the empty niche-structure provided by the permissive medium would be instantaneous, and no structure "deeper" than the permissive medium is called for. This idea seems to resonate with the idea of the quantum potential of Bohm [7], but without the constraints of mathematical formalism, at least in that it could be said to be a self-organizing process of the underlying field that lays down a guide or template. Since there is no reason why the empty niche structures of the type suggested could not communicate through the whole permissive medium, it would effectively involve the "whole field", and carry information about any experimental arrangement in which a particle would find itself. 
Thus the niche-particle is trapped in a niche-structure selected by current reality. This amounts to the current experimental setup. If the experimental setup were to be changed, (in particular, the orientation or presence of slits, arms or mirrors) then the empty niche-structure, which defines the route the particle will take, must adjust to this changed conformation. Since time arises out of changes in the occupied nichestructure, not the empty niche-structure "beneath" it, any change in the empty nichestructure would always be perceived from above as instantaneous. There would be no constraint on the distance over which an empty niche-structure could develop; only that it should be maximally economical in an energetic (informational) sense. Thus it would conform to the principle of least action.

An approach taken by many investigators in the field of Quantum Interactions is to take an understanding of the quantum formalism, and apply it to problems resistant to other techniques. The approach taken in this paper was different. Revealed in the genetic interaction map was an image of "the organism" as a functional network, and then as an occupied niche. Reduction allowed the development of a framework, with quantum particles visualized as occupied niches, evolving upon an empty niche structure, in turn, selected within the permissive medium constituting Planck-scale interactions. The framework is simple, has no algebraic content, and may help towards a better understanding of quantum interactions, and to further the interpretation of quantum theory generally.

Acknowledgements. I am grateful for helpful discussions with Vic Norris, Dominic Widdows, Peter Wittek, Terry Robinson and Mike Halsall.

\section{References}

1. Costanzo, M., and 52 others. The genetic landscape of a cell. Science 327, 425 431 (2010)

2. Benzer, S. On the topology of the genetic fine structure. Proc. Natl. Acad. Sci. USA 45, 1607-1620 (1959)

3. Costanzo, M., Baryshnikova, A., Myers, C.L., Andrews, B., and Boone, C. Charting the genetic interaction map of a cell. Curr. Op. Biotechnol. 22, 1-9 (2010)

4. Bray, D. Wetware: a computer in every living cell. Yale University Press (2009)

5. Peter, S., Veloz, T., and Dittrich, P. Feasibility of Organizations. Membrane Computing, Lecture Notes in Computer Science 6501, 325-337. (2011)

6. Jeong, H., Tombor, B., Albert, R., Oltvai, Z.N., and Barabasi, A.L. The largescale organization of metabolic networks. Nature 407, 651-654. (2000)

7. Bohm, D., Hiley, B.J., and Kaloyerou, P.N. An ontological basis for the quantum theory. Physics Reports 144, 321-375. (1987) 